\title{
Análisis de contenido semántico: evolución del discurso modal de la inmigración en la prensa española
}

Semantic content analysis: the evolution in modal discourse on immigration in the Spanish press

\author{
Javier Álvarez Gálvez \\ Universidad Loyola Andalucía \\ Universidad de Sevilla \\ javieralvarezgalvez@gmail.com; jalvarez@uloyola.es (ESPAÑA)
}

Recibido: 09.122011

Aceptado: 19.12.2012

\section{RESUMEN}

Con objeto de obtener una mayor comprensión del cambio de opiniones y actitudes de los españoles hacia los inmigrantes (Cea D'Ancona, 2004, 2007), este estudio analiza la evolución del discurso mediático de la inmigración en los editoriales de tres periódicos españoles: El País, El Mundo y La Vanguardia (2000-2008). Para ello, se emplea una novedosa técnica de análisis de contenido semántico que nos permite detectar desplazamientos significativos del espacio discursivo de la inmigración. Los resultados de este trabajo muestran claros indicios de la evolución del discurso de la inmigración hacia temas relacionados con la inseguridad y la demanda de soluciones políticas.

\section{PALABRAS CLAVE}

Inmigración, actitudes, discurso, análisis de contenido semántico.

\section{ABSTRACT}

In order to obtain a better understanding of the change of opinions and attitudes of the Spanish toward immigrants (Cea D'Ancona, 2004, 2007), this 
study analyzes the evolution of the media discourse referring to immigration in three Spanish newspapers: El País, El Mundo and La Vanguardia (2000-2008). In this work, a novel technique of semantic content analysis is applied to detect significant shifts in this discourse. The results show clear evidences of the evolution of the discourse regarding the immigration and its association with security issues and the demand for political solutions.

\section{KEYWORDS}

Immigracion, attitudes, discourse, semantic content analysis.

\section{INTRODUCCIÓN}

El incremento de la presencia de inmigrantes de los últimos años ha fomentado el surgimiento de opiniones y actitudes contrarias a la inmigración, tanto en España como en muchos otros países del contexto europeo. El discurso de los medios de comunicación ha desempeñado un papel fundamental en dicho cambio de actitudes. Así, son numerosos los estudios en nuestro país que afirman la importancia de los medios de cara a la (re)producción de actitudes hacia la inmigración. Entre otros: van Dijk, 1991; Checa y Escobar, 1996; Granados, 1998; Rodrigo Alsina, 1999; Valles, Cea e Izquierdo, 1999; Santamaría, 2002; Cea D’Ancona, 2004; Lorite, 2004; Igartua, Muñiz y Cheng, 2005; Casero, 2006.

Este trabajo de investigación estudia la evolución del discurso mediático de la inmigración con objeto de obtener una mayor comprensión del cambio de opiniones y actitudes de los españoles hacia los inmigrantes. Para ello, se emplea una novedosa técnica denominada modality analysis (Roberts et al. 2010), diseñada para analizar el cambio discursivo de los actores sociales en función del estudio de formas modales del lenguaje. Partiendo de la premisa de que todo sistema social es justificado en base al uso discursivo de las cláusulas modales mediante las cuales los actores sociales delinean qué acciones son posibles, imposibles, inevitables o contingentes dentro de un determinado contexto, nuestro trabajo de investigación se centra en analizar los posibles cambios en la modalidad lingüística del discurso de la inmigración en la prensa española durante el período 1999-2008.

Como ya se ha señalado en otros trabajos de investigación (Roberts, 2008; Roberts et al., 2010), las formas verbales modales (poder, tener que, deber de, estar obligado a, ser capaz de, etc.) suelen aparecer en los textos como un recurso discursivo mediante el cual la fuente del texto (es decir, el hablante o el autor) expresa la intención de un determinado verbo de la oración (en su modo infinitivo). Generalmente, estas formas modales tenderán a ir acompañadas por razones o justificaciones que explican por qué dicho sujeto puede, debe, está obligado a, etc. actuar de una manera particular. Pudiendo asimismo dar lugar 
a distintas modalidades que reflejan diferentes formas culturales de entender «qué son capaces de hacer los actores sociales» (modalidad del logro), «qué les está permitido hacer» (modalidad de permisión), «qué necesitan hacer» (modalidad de necesidad) o «qué están obligados a hacer» (modalidad de obligación). Modalidades que, teniendo su fundamentación teórica en las formas de socialización de Simmel, definen «qué razones» son usadas por los actores sociales para legitimar «qué acciones».

\section{LA JUSTIFICACIÓN DISCURSIVA DEL RECHAZO}

La inversión de los flujos migratorios en las últimas dos décadas ha provocado un significativo cambio en las actitudes de los españoles hacia el fenómeno de la inmigración. En efecto, recientes estudios centrados en el análisis de las opiniones y las actitudes de los españoles hacia la inmigración, ponen de manifiesto que la percepción del fenómeno de la población española ha cambiado sustancialmente entre las últimas dos décadas (Díez Nicolás y Ramírez Lafita, 2001; Cea D'Ancona, 2004, 2007; Cea D'Ancona y Valles Martínez, 2008, 2009). De hecho, el actual fenómeno de la inmigración es ya considerado por los españoles como uno de los problemas principales del país, seguido muy de cerca de los problemas de paro, economía, inseguridad ciudadana, terrorismo y vivienda. Una tónica que lleva repitiéndose desde algunos años atrás, y puede apreciarse en los barómetros del CIS. Llegando, en los meses de septiembre y octubre de 2006, a considerarse la inmigración el principal problema de España. Un fenómeno que coincidió con un momento de gran revuelo mediático en torno al tema de la inmigración, sobre todo a consecuencia de la denominada «crisis de los cayucos de Canarias $\rangle^{1}$. Asimismo, se hace patente el alto grado de concordancia entre la opinión de los españoles respecto al fenómeno de la inmigración y el tratamiento mediático del mismo. Como ya se mencionara en otros trabajos de investigación (Cea D'Ancona, 2007), la inmigración de ha convertido en uno de los temas principales de la agenda de los medios informativos.

Evidentemente, no podemos achacar este novedoso cambio en las opiniones y las actitudes de los españoles exclusivamente a la acción informativa de los medios de comunicación, ya que en realidad existe un cambio demográfico de fondo (tanto en España como en todo el contexto europeo) y, por consiguiente, un aumento en la percepción de la presencia de inmigrantes. No obstante, distintas investigaciones reconocen la importancia de los medios como formadores de la opinión pública en actitudes xenófobas hacia el colectivo inmigrante (Cea D’Ancona, 2004, 2007; Igartua, Muñiz y Cheng, 2005; Valles, Cea e Izquierdo, 1999; van Dijk, 1994, 1997, 2003), una lógica consecuencia del tratamiento

${ }^{1}$ Hay que tener en cuenta que a finales de verano de 2006 y en los meses posteriores las noticias sobre inmigración tenían una frecuencia de aparición diaria. Además, el tratamiento de los acontecimientos se volvió mucho más problemático. Lo que posibilitó un aumento de la llamada «psicosis de la invasión» (Valles, Cea e Izquierdo, 1999; 43). 
negativo que, por lo general, suele recibir la información sobre inmigración. Ante las imágenes de «invasión», «avalancha» u «oleada» de inmigrantes transmitidas por los medios en los últimos años, el fenómeno comienza a percibirse como algo incómodo para los autóctonos, empiezan a surgir recelos. La imagen de la «llegada incontrolada de pateras a las costas españolas», las «bandas latinas» (Latin Kings, Ñetas, Dominican Don't Play...), los «asaltos de casas llevados a cabo por bandas organizadas de Europa del Este», la «competencia desleal del pequeño empresario chino», el «aumento en las tasas de delincuencia», el «tráfico de drogas», la «prostitución», etc., son, entre otros, algunos de los atributos que configuran la imagen global de la inmigración —o, más correctamente, el marco desde el que la inmigración es socialmente evaluada como fenómeno- - No es de extrañar entonces que de cara a la opinión pública el fenómeno se problematice (Vecina, 2012).

Aunque en España, a diferencia de otros países europeos, la inmigración no es excesivamente maltratada a través de los medios de comunicación de masas (van Dijk, 2003), existe toda una línea discursiva de las élites políticas acerca de los pros y los contras de la inmigración. De hecho hablar de inmigración siempre saca a luz un conocido corpus temático. Hablar de la inmigración en términos positivos implica hacer referencia al «enriquecimiento de la cultura», a los «beneficios económicos», al «aumento de la natalidad», etc. Hablar en negativo, al contrario, trae a colación el tema de la «delincuencia», la «percepción en demasía» del número de inmigrantes en el país, los «problemas de integración», «problemas de desempleo», etc. En definitiva, distintos discursos públicos de la inmigración (político, periodístico, académico,...), que hablan acerca de los beneficios y/o problemas que el fenómeno conlleva, y que, usualmente, tiene a los medios de comunicación como fuente principal de distribución. Por consiguiente, debemos tener en cuenta que gran parte del conocimiento que tenemos acerca de la sociedad y el mundo que nos rodea, incluidas todas las informaciones que tenemos acerca de la inmigración, proviene de los medios de comunicación de masas (Luhmann, 2000). Estos son los vehículos a través de los cuales se construyen cogniciones socialmente compartidas y formas de interpretar la realidad (D’Adamo, García y Freidenberg, 2007) que, en ciertas ocasiones, actuarán como justificaciones sobre el modo en el que los autóctonos se interrelacionan con los inmigrantes. Esto es, como argumentos que legitiman el despliegue de determinadas líneas discursivas y, por lo tanto, acciones hacia los inmigrantes.

Los medios de comunicación operan en el «ámbito público» y, del mismo modo, son públicamente regulados. Toman información del contexto (social, político, económico y cultural) y la procesan para posteriormente transmitirla como producto final que vuelve al medio del cual fue extraído. Los medios de comunicación de masas, como productores y distribuidores de contenidos de carácter simbólico (y, en definitiva, de conocimiento) tienen capacidad para construir y reinterpretar la realidad (Luhmann, 2000). Del mismo modo que las personas definimos las situaciones y categorizamos a otras personas en la vida cotidiana (Goffman, 1974), los medios de comunicación, también colaboran en el proceso 
de definición social y etiquetado. De hecho, los medios de comunicación de masas podrían ser considerados como extensiones de las propias facultades sensoriales del individuo (McLuhan, 1964). Desde este punto de vista, los medios de comunicación de masas, no sólo transmitirían la información del entorno (local o global) en el que se desenvuelven, sino que, incluso podrían crear y/o modificar corrientes de opinión bajo circunstancias determinadas de crisis o momentos de incertidumbre (Ball-Rokeach y DeFleur 1976; Ball-Rokeach, 1985), como, por ejemplo, los vividos en los últimos años. De ahí la importancia de los mismos en las sociedades contemporáneas y, en nuestro caso, de cara a la definición del fenómeno migratorio actual y justificación del rechazo hacia los inmigrantes.

Probablemente, el mejor modo de definir los «medios de comunicación de masas» sea centrarnos en las funciones que estos desempeñan. Siguiendo a D’Adamo, García Beaudoux y Freidenberg (2000), podríamos señalar seis funciones básicas de los medios de comunicación que serían las siguientes: 1) ofrecer información; 2) correlación, cuyo objetivo es explicar, interpretar y comentar el significado de los acontecimientos; 3) ofrecer diversión y entretenimiento; 4) persuadir, como un modo de generar tendencias en la opinión pública; 5) servir al sector económico a través de la función publicitaria y; 6) transmisión de cultura (socialización), aunque no constituya, en principio, un objetivo deliberado de los medios. Desde este punto de vista, los medios no sólo ofrecerían información, sino que serían capaces de explicar las causas y las consecuencias de los acontecimientos, interpretar los hechos, establecer significados, persuadir acerca de los posibles cauces de acción social y, en última instancia, transmitir cultura y socializar. Por consiguiente, a pesar de que en ciertas ocasiones se sobreestime la capacidad de los medios de cara a la generación o manipulación de la opinión pública, no podemos negar la preeminencia de estos a la hora de definir los acontecimientos y, por lo tanto, categorizar a las personas dentro de un contexto socio-cultural determinado (Álvarez Gálvez, 2009).

En efecto, en el caso de la inmigración (como, por supuesto, en muchos otros temas), los medios se sitúan en un lugar privilegiado a la hora de definir el marco de referencia a partir del cual los inmigrantes serán socialmente evaluados ${ }^{2}$. Por un lado, establecen la agenda de temas posibles sobre qué pensar (McCombs, 2004), mientras que, por otro, organizan y establecen toda una gramática de significado sobre los temas en cuestión (este tema será desarrollado a continuación). Asimismo, debemos pensar que gran parte de los conocimientos sobre la inmigración que tienen los españoles proviene de los medios de comunicación, ya que aunque actualmente se tiene una imagen más real debido a la mayor presencia y proximidad de los inmigrantes, en ausencia de relaciones cercanas, el encuadre de los medios sigue predominando. Si no, ¿cómo sería posible que personas autóctonas que nunca han tenido ni contacto ni relación (proximidad) con las

2 Siguiendo a Goffman, se podría decir que el marco de referencia primario es el material, creado socialmente, a partir del cual conferimos sentido a nuestro mundo. Un «esquema interpretativo» que permite a los individuos «situar, percibir, identificar y etiquetar un número aparentemente infinito de sucesos concretos definidos en sus términos» (1974: 23). 
distintas tipologías de inmigrantes residentes en España puedan tener unas mismas ideas sobre cada una de éstas? Indudablemente, aunque los medios de comunicación no sean deliberadamente los creadores de la imagen del Otro, puesto que: $1^{\circ}$ ) dicha imagen ya se encuentra implícita en el discurso de dominación histórico en el que somos socializados (Said, 1990); y $2^{\circ}$ ) de nuestra experiencia directa con el Otro en el contexto. Podemos pensar que en ausencia de un contacto real y continuado con el Otro, será la imagen ofrecida por los medios la que configure - hasta cierto punto- nuestro marco de referencia y los presupuestos discursivos sobre los que se sustentará nuestra imagen de la inmigración. Lo que claramente representa un problema si tenemos en cuenta la gran reducción de la realidad que se lleva a cabo al encuadrar una minúscula porción de la misma. De hecho, el proceso de pasar la amplia diversidad de la realidad por el minúsculo filtro de los medios es lo que conduce, de un modo inevitable, a la pérdida de información ${ }^{3}$. Una pérdida de información sobre los Otros, que a veces acaba generando una representación distorsionada — cuando no falseada — de la realidad de estos y, en último término, prejuicios y estereotipos estructurantes y persistentes en el tiempo (Santamaría, 2002). Prejuicios y estereotipos mediáticos que acaban por justificar el aumento del rechazo hacia los inmigrantes, ya que sirven de razones de cara a la legitimación de posturas contrarias a la inmigración.

\section{MODALIDAD LINGÜÍSTICA Y DISCURSO}

Teniendo en cuenta el aumento del rechazo hacia la inmigración que se ha producido en los últimos años (Cea D'Ancona, 2004, 2007; Cea D'Ancona y Valles Martínez, 2008, 2009) y, por consiguiente, de la existencia de un cambio discursivo de fondo acerca de los temas relacionados con la inmigración, este trabajo se centrará en el análisis de dicho cambio. Partiendo de la premisa de que todo sistema social es justificado en base al uso discursivo de las cláusulas modales mediante las cuales los actores sociales delinean qué acciones son posibles, imposibles, inevitables o contingentes dentro de un determinado contexto, nuestro trabajo de investigación analiza los posibles cambios en la modalidad lingüística del discurso de la inmigración en la prensa española durante el período 1999-20084.

Así, se pone a prueba una tipología específica de análisis de contenido semántico denominada «modality analysis» diseñada para el cambio del discurso modal. Esta tipología de análisis semántico tiene como ventaja el hecho

${ }^{3}$ De hecho, antes de juzgar la labor de los profesionales del periodismo, resulta conveniente reflexionar sobre las dificultades de dicho oficio y, por supuesto, las limitaciones propias del sistema productivo de conocimiento en el que está inserto (Rodrigo, 2005).

${ }^{4}$ La opción de los medios escritos se debió principalmente a criterios estratégicos. Por un lado, resultaba más fácil acceder a la información textual a través de los buscadores de los websites de los diarios empleados en nuestro estudio. Por otro, no creemos que puedan existir muchas diferencias a nivel discursivo entre medios visuales y escritos ya que estamos tratando con el mismo discurso social. 
de que nos permite trabajar con una gramática de significado $\mathrm{y}$, a diferencia de otras tipologías analíticas (análisis de contenido temático o de redes), posibilita el análisis del cambio discursivo. Es decir, no sólo se analiza la aparición de determinados elementos en el texto, sino también sus relaciones internas y su evolución temporal. Dicho análisis se basa en la premisa de que es posible localizar diferencias culturales fundamentales en el uso discursivo que los participantes de una misma cultura hacen de los verbos modales auxiliares (Roberts et al. 2010: 3). Diferencias en el uso modal del lenguaje que pueden ser localizadas en el «espacio discursivo» de las culturas. El «modality analysis» permite, concretamente, comparar cómo personas procedentes de distintas culturas difieren en sus formas de comprender y justificar por qué ciertas acciones pueden ser llevadas a cabo (o no) en el marco de un determinado contexto cultural. En este sentido, será habitual observar que el uso de los verbos modales auxiliares (por ejemplo, ser capaz de, tener que, estar obligado a, tener permiso para, etc.) se encuentre acompañado de las razones o argumentos mediante la/os cuales los actores sociales justifican que acciones son posibles, imposibles, inevitables o contingentes (no-inevitables). En definitiva, razones que nos indican qué acciones pueden ser llevadas a cabo por qué motivos en el contexto cultural en el que se enmarca la acción social y, que en último término, exteriorizan la dirección del discurso público y la intencionalidad de los actores (Roberts et al., 2010).

A nivel discursivo (sea en el habla o en el texto), los verbos modales auxiliares son empleados como un recurso a través del cual la fuente del texto (esto es, el hablante o el autor del texto) transmite la intención del sujeto de la oración o la suya propia. En la oración el verbo modal auxiliar irá generalmente acompañado del verbo principal, en su forma de infinitivo, cuyo significado será modificado por el verbo modal auxiliar que lo precede. Paralelamente, este tipo de construcciones modales irán acompañadas por, como decimos, razones o argumentos que explican por qué este sujeto puede, debe, está obligado a,... actuar de un modo específico. Así, siguiendo los presupuestos teóricos de Roberts et al. (2010), creemos que esas razones reflejan creencias y valores que probablemente diferirán entre distintos contextos culturales. Aunque, como indican Roberts et al. (2010: 4), «el modality analysis puede ser empleado para hacer explícitas "qué razones" son usadas para legitimar "cuáles actividades" en diferentes contextos culturales y, asimismo, para hacernos una idea de las diferencias culturales a través del modo en cómo las motivaciones de las personas son discursivamente mantenidas», presuponemos también la utilidad de esta técnica de análisis lingüístico de cara al estudio del cambio de las formas modales del lenguaje en contextos en proceso de transformación social (como en el caso actual de la inmigración). Esto es, aunque el potencial metodológico de esta técnica se encuentra en la posibilidad comparativa de distintas modalidades discursivas de los diferentes contextos culturales, también presenta utilidad para el análisis del cambio discursivo modal dentro de una misma cultura, como ya se ha probado en otros trabajos de investigación (Roberts, Popping y 
Pan, 2009). Es por eso que nuestra intención se dirige a analizar la evolución de este discurso en la prensa española, ya que, lógicamente, presuponemos la existencia de un cambio discursivo mediático que puede estar relacionado con el aumento del rechazo hacia los inmigrantes en los últimos años.

Hasta el momento hemos definido el «modality analysis» como una metodología de análisis textual que permite realizar inferencias acerca de la modalidad lingüística de un lenguaje enmarcado en un contexto cultural específico. Ahora bien ¿qué es lo que entendemos por modalidad lingüística? De un modo simple se podría decir que una modalidad lingüística es un lenguaje de motivaciones (Roberts, 2008). Un lenguaje que exterioriza las motivaciones de los actores, sus intenciones y propósitos de cara a la consecución de ciertos fines o metas. En este sentido, estaríamos hablando de un lenguaje de carácter persuasivo mediante el cual los actores sociales expresan la posibilidad, imposibilidad, inevitabilidad o contingencia de sus proyectos, la finalidad de sus acciones.

Pensemos en las siguientes oraciones modales: Por $x$ razones, Isabel puede actuar; Isabel no puede actuar; Isabel rehúsa actuar; Isabel tiene que actuar; Isabel debe actuar; Isabel quiere actuar; Isabel está obligada a actuar; etc. Como vemos en este ejemplo, las oraciones modales presentan un verbo modal auxiliar (poder, no poder, deber, tener que, querer, estar obligado a, etc.) junto a una forma verbal en infinitivo (en nuestro caso: actuar). Un verbo en infinitivo cuyo significado es modificado, o mejor dicho reorientado, por las distintas formas modales (puede, no puede, rehúsa, tiene que, debe, quiere, está obligada a actuar). Sin embargo, entendiendo la modalidad lingüística como un lenguaje de motivaciones dirigidas a determinados fines, podemos suponer que detrás de las intenciones del sujeto de la oración (Isabel) o detrás de las propias intenciones de la fuente del mensaje (el emisor) existen ciertos argumentos ( $x$ razones) que son los que justifican la intencionalidad de los actores.

En este sentido, toda modalidad lingüística estará formada por dos elementos fundamentales. Por un lado la forma modal (verbo modal auxiliar + forma verbal en infinitivo) y, por otro, las razones que justifican la intención del sujeto o fuente del mensaje. Así, en función de la intencionalidad de la persona identificaríamos dos tipos de modalidades: a) la modalidad relacionada a personas (sujeto de la oración); y b) la modalidad relacionada al contexto (fuente del mensaje). Pensemos en estas dos sentencias modales:

[A] Porque piensa que el país no va bien, Isabel quiere gobernar.

[B] Porque el país no va bien, Isabel debe gobernar.

Como se puede observar, estamos ante dos sentencias modales cuya estructura muestra unas razones («el país no va bien») que justifican ciertas intenciones. Ahora bien, si la modalidad relacionada a personas (en la sentencia A) expresa las intenciones del sujeto de la oración (Isabel quiere gobernar), la modalidad relacionada al contexto (sentencia B) presenta las intenciones de la fuente del mensaje (Isabel debe gobernar), unas intenciones que, como se puede apreciar, van más allá de los deseos del sujeto de la oración. En la sentencia A, 
Isabel cree posible cambiar el país y por eso quiere (o tiene la intención de) cambiarlo. En la sentencia B, no importa lo que Isabel quiera o deje de querer, sino que lo relevante, en este caso, es la creencia de la fuente del texto acerca de la idoneidad de Isabel como gobernante de cara al cambio del país. Una opinión que expresa inevitabilidad (debe gobernar) y que, como decimos, va más allá de las propias intenciones de Isabel.

Aunque, en principio, la identificación de la modalidad lingüística no parece representar un gran problema, en la práctica, podemos encontrar ciertas dificultades. Roberts et al. (2010) indican los siguientes pasos en la identificación de la modalidad lingüística en el texto: 1) localización de las formas verbales de carácter modal que expresen intencionalidad; 2) si no las hay, localizar partes del texto que puedan ser legítimamente transformadas en formas modales; 3) comprobar la existencia de intencionalidad; y en último lugar, 4) comprobar que el sujeto semántico de la oración sea una persona. Aunque es habitual que la modalidad lingüística aparezca en la forma típica [verbo modal + verbo infinitivo], en ocasiones, podemos encontrar construcciones gramaticales que en forma pasiva (por ejemplo, «Por $\mathrm{X}$ razones, $\mathrm{Y}$ debe ser gobernado por $\mathrm{Z}$ ») transmiten la misma intencionalidad y, por consiguiente, pueden ser legítimamente transformadas ( $«$ Por $\mathrm{X}$ razones, $\mathrm{Z}$ debe gobernar $\mathrm{Y}$ ). Asimismo, también es posible encontrar formas modales que, al no estar justificadas por determinadas razones, no constituyen una verdadera modalidad. Incluso podríamos hallar casos en los que exista una razón y una forma modal pero que no presenten un sujeto semántico humano, lo que anularía la existencia de racionalidad humana $\mathrm{y}$, en definitiva, de intenciones. Por ejemplo, decir «el microondas no puede calentar» no transmite ninguna intencionalidad o motivación, ni siquiera aunque encontráramos algunos argumentos que justificaran la imposibilidad del microondas para llevar a cabo la acción de calentar.

Como indican Roberts et al. (2010: 8), «una cláusula modal siempre transmite, a través de su fuente, un juicio sobre las motivaciones del sujeto semántico de la cláusula modal». Así las sentencias modales nunca serán descriptivas o diagnósticas, sino que expresarán una imputación de intencionalidad mediante el uso de formas modales susceptibles de expresar posibilidad (querer, esperar, intentar, poder, ser capaz de,... actuar), imposibilidad (no poder, no ser capaz de, ... actuar), inevitabilidad (tener que, deber, estar obligado a,... actuar) o contingencia (ser capaz de no, no estar obligado a,... actuar).

A diferencia de otros tipos de análisis textuales, el modality analysis no se centra en el estudio de la aparición de palabras u oraciones sobre un tema determinado (como, por ejemplo, el análisis de contenido temático), sino en el análisis de gramáticas semánticas (semantic grammars), bloques de texto semánticamente interrelacionado. Por consiguiente, en lugar de analizar temas o categorías, este tipo de estudios están enfocados al análisis de las relaciones 
semánticas entre determinados elementos del texto (Roberts, 2000). Así, la gramática semántica del modality analysis presentaría dos partes fundamentales:

$$
\text { RAZÓN }\{\text { ARGUMENTOS }\}+\text { FORMA MODAL }\left\{\begin{array}{c}
\text { POSIBLE } \\
\text { IMPOSIBLE } \\
\text { INEVITABLE } \\
\text { CONTINGENTE }
\end{array}\right\}
$$

En nuestro trabajo de investigación, partiendo de la premisa de la transformación de la modalidad lingüística del discurso de la inmigración que justifica los actuales cambios opinático-actitudinales hacia la inmigración de los últimos años, se realizó un análisis de la modalidad lingüística (modality analysis) del discurso de la prensa en tres diarios españoles. Lógicamente, el discurso extraído de tan sólo tres diarios carecía de representatividad, ya que ni estos eran los únicos medios informativos de comunicación, así como tampoco los más usados (como, por ejemplo, la televisión). Sin embargo, podemos suponer que el discurso mediático como producto cultural estará representado más o menos del mismo modo en los diferentes medios. Por consiguiente, en este estudio la unidad de análisis sería la cláusula modal anidada en artículos editoriales ${ }^{5}$.

En este caso, la cláusula modal presentaría una estructura de significado (gramática semántica) de tres partes: 1) una razón (cultural, económica, política, de bienestar o de seguridad); 2) una forma modal (posible, imposible, inevitable o contingente); y 3) un sujeto semántico de la oración (español, miembro de la Unión Europea o Inmigrante). La gramática semántica extraída presentaría el siguiente significado: «Hay una razón \{cultural, económica, política, de bienestar o de seguridad\} por la que algo puede ser \{posible, imposible, inevitable o contingente\} para un sujeto semántico \{español, europeo

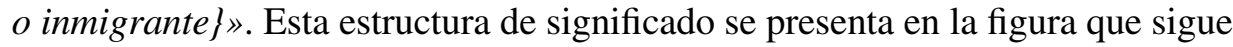
a continuación:

RAZÓN $\left\{\begin{array}{c}\text { CULTURAL } \\ \text { ECONÓMICA } \\ \text { POLÍTICA } \\ E^{\circ} \text { BIENESTAR } \\ \text { SEGURIDAD }\end{array}\right\}+$ FORMA MODAL $\left\{\begin{array}{c}\text { POSIBLE } \\ \text { IMPOSIBLE } \\ \text { INEVITABLE } \\ \text { CONTINGENTE }\end{array}\right\}+$ SUJETO SEMÁNTICO $\left\{\begin{array}{c}\text { ESPAÑOL } \\ \text { MIEMBRO UE } \\ \text { INMIGRANTE }\end{array}\right\}$

\subsection{Metodología del estudio y datos empleados}

En total se seleccionarían 1129 cláusulas modales anidadas en una población de 528 editoriales sobre inmigración extraídos de tres diarios españoles:

${ }^{5}$ Se tomarían artículos editoriales porque se pretendía analizar la intencionalidad en el texto. De ahí que se seleccionaran artículos de opinión, no descriptivos. 
El País, El Mundo y La Vanguardia ${ }^{6}$ (1999-2008). En este caso, el discontinuo tratamiento del tema de la inmigración a nivel editorial nos permitió trabajar con la población completa de artículos editoriales ${ }^{7}$.

Teniendo en cuenta la anidación de las cláusulas modales, así como la disposición de los editorialistas a hacer hincapié en la coherencia del mensaje que intentan transmitir, los artículos editoriales tenderán a repetir las mismas formas modales (y sus justificaciones) en su interior. Un efecto de reagrupamiento de las cláusulas modales que se presenta en las frecuencias de celda. De ahí que, como ya se hiciera en trabajos anteriores (Roberts, Popping y Pan 2009), se emplearía un modelo multinivel para afrontar este problema y, al mismo tiempo, probar nuestras hipótesis sobre el modo de asociación de nuestras variables de primer nivel con respecto a otras variables contextuales (ej. tiempo lineal) de segundo nivel (Davidian y Giltinan, 1995; Vonesh y Chinchilli, 1997). Es decir, sobre la existencia de una transformación a nivel discursivo en el tiempo (en la primera década del nuevo milenio). El modelo multinivel utilizado en este análisis es el siguiente (Pinheiro y Bates, 1995):

$$
\begin{gathered}
\log \left(\mathrm{m}_{\mathrm{ijk}}+\Delta\right)=\lambda+\lambda_{i}^{M}+\lambda_{j}^{R}+\lambda_{k}^{P}+l \lambda^{L}+\lambda_{i j}^{M R}+\lambda_{i k}^{M P}+l \lambda_{i}^{M L}+\lambda_{j k}^{R P}+ \\
l \lambda_{j}^{R L}+l \lambda_{k}^{P L}+\lambda_{i j k}^{M R P}+l \lambda_{i j}^{M R L}+l \lambda_{i k}^{M P L}+l \lambda_{j k}^{R P L}+l \lambda_{i j k}^{M R P L}
\end{gathered}
$$

En este modelo, $\mathrm{m}_{\mathrm{ijk}}$ es la frecuencia esperada de $i$ formas modales (codificadas como indicadoras de: posibilidad, imposibilidad, inevitabilidad o contingencia), $j$ razones o justificaciones asociadas (codificadas como: culturales, económicas, políticas, de seguridad, de bienestar) y $k$ sujetos semánticos de la cláusula modal (sean estos españoles, miembros de la UE o inmigrantes), los tres elementos básicos que componen la gramática semántica de las cláusulas modales. La variable $l$ será el tiempo lineal en incrementos de un año desde $-4,5$ hasta 4,5 para el período 1999-2008, y las $\lambda$ representan los efectos de interacción entre las distintas variables del modelo. Además, siguiendo a Agresti (1990: 250), para salvar el problema de las tablas de contingencia incompletas, añadimos el valor $\Delta=10^{-8}$ a cada una de las celdas de nuestra tabla. Asumimos

${ }^{6}$ La razón de optar por estos tres periódicos respondió en cierto modo a criterios estratégicos. La posibilidad de tener acceso a las ediciones impresas a través de Internet para el período de estudio y la posibilidad de emplear los buscadores a modo de filtro fueron puntos determinantes a la hora de realizar la elección de dichos diarios. Por otra parte, no se establecieron diferencias en función de la ideología de los tres diarios, ya que al ser un estudio exploratorio se pretendía captar el cambio general en el discurso y no las especificidades discursivas de los mismos.

${ }^{7}$ Los artículos serían clasificados como «editoriales sobre inmigración» si: 1) Pertenecían a la sección editorial del periódico; 2) Hacían referencia a la inmigración como tema principal; 3) Contenían al menos una sentencia modal en los tres primeros o últimos párrafos del artículo (ya que generalmente es el lugar donde el autor del texto suele expresar su opinión y, por consiguiente, sus intenciones). 
que el error tiene una distribución normal sobre cero y que las frecuencias de celda observadas tienen una distribución Poisson.

Dados nuestros 528 editoriales más los cuatro niveles para las variables de nuestro estudio (forma modal, razón y sujeto semántico), la tabla de este modelo tendría $9.180(4 \times 5 \times 3 \times 528)$ celdas, demasiadas para que nuestro modelo adquiriera cierta potencia de cara a la realización de inferencias estadísticas acerca de la gran cantidad de efectos que se intentan estimar. Por este motivo el número de celdas se reduciría a $4.224(2 \times 2 \times 2 \times 528)$ permitiendo, a pesar de la dificultad mantenida, el análisis separado de cada una de las combinaciones de nuestras variables en el modelo (forma modal + razón + sujeto semántico). Así el modelo log-lineal multinivel anteriormente expuesto se ajustó a 60 tablas de 4.224 celdas cada una.

\section{LA MODALIDAD LINGÜÍSTICA DE LA INMIGRACIÓN EN LA PRENSA}

En la tabla 1 se muestran los porcentajes de cláusulas modales asociadas a razones y sujetos semánticos que finalmente se codificaron. Como se puede apreciar en estos datos, las formas modales de inevitabilidad predominan para sujetos españoles y europeos, con porcentajes por encima del $50 \%$, mientras que las formas de posibilidad son más frecuentes para los sujetos inmigrantes. Las razones de seguridad son las predominantes en sujetos semánticos españoles (33\%) y europeos (49\%), mientras que las económicas son las más frecuentes para sujetos inmigrantes. En última instancia, hay que resaltar el mayor peso de las sentencias modales referidas a sujetos semánticos españoles.

Tabla 1. Porcentajes de sentencias modales asociadas a razones y sujetos $(\mathbf{m} \times \mathbf{r} \times \mathbf{p})$ que fueron codificadas para el análisis

\begin{tabular}{|c|c|c|c|c|c|c|c|}
\hline & \multirow{2}{*}{$\begin{array}{l}\text { Forma } \\
\text { modal } \\
\text { auxiliar }\end{array}$} & \multicolumn{6}{|c|}{ Razón o argumento asociado } \\
\hline & & Cultural & Económico & Político & Seguridad & Bienestar & Total \\
\hline \multirow[t]{5}{*}{ Español } & Posible & $0,3 \%(2)$ & $1,2 \%(8)$ & $8,1 \%(54)$ & $3,7 \%(25)$ & $2,2 \%(15)$ & $15,6 \%(104)$ \\
\hline & Imposible & $3,1 \%(21)$ & $2,7 \%(18)$ & $7,6 \%(51)$ & $8,1 \%(54)$ & $5,5 \%(37)$ & $27,1 \%(181)$ \\
\hline & Contingente & $0,1 \%(1)$ & $0,6 \%(4)$ & $1,2 \%(8)$ & $0,0 \%(0)$ & $0,1 \%(1)$ & $2,1 \%(14)$ \\
\hline & Inevitable & $3,9 \%(26)$ & $5,7 \%(38)$ & $11,7 \%(78)$ & $21,0 \%(140)$ & $12,9 \%(86)$ & $55,2 \%(368)$ \\
\hline & Total & $7,5 \%(50)$ & $10,2 \%(68)$ & $28,6 \%(191)$ & $32,8 \%(219)$ & $20,8 \%(139)$ & $100,0 \%(667)$ \\
\hline \multirow[t]{5}{*}{ European } & Posible & $0,5 \%(1)$ & $2,7 \%(5)$ & $5,9 \%(11)$ & $10,8 \%(20)$ & $0,5 \%(1)$ & $20,5 \%(38)$ \\
\hline & Imposible & $2,2 \%(4)$ & $3,2 \%(6)$ & $6,5 \%(12)$ & $10,8 \%(20)$ & $1,1 \%(2)$ & $23,8 \%(44)$ \\
\hline & Contingente & $0,0 \%(0)$ & $0,5 \%(1)$ & $1,1 \%(2)$ & $1,1 \%(2)$ & $0,0 \%(0)$ & $2,7 \%(5)$ \\
\hline & Inevitable & $4,9 \%(9)$ & $4,3 \%(8)$ & $10,8 \%(20)$ & $26,5 \%(49)$ & $6,5 \%(12)$ & $53,0 \%(98)$ \\
\hline & Total & $7,6 \%(14)$ & $10,8 \%(20)$ & $24,3 \%(45)$ & $49,2 \%(91)$ & $8,1 \%(15)$ & $100,0 \%(185)$ \\
\hline
\end{tabular}




\begin{tabular}{ccccccc}
\hline $\begin{array}{c}\text { Forma } \\
\text { modal } \\
\text { auxiliar }\end{array}$ & Cultural & Económico & Político & Seguridad & Bienestar & \multirow{2}{*}{ Total } \\
\cline { 2 - 7 } & & & & & & \\
\hline Inmigrante Posible & $2,2 \%(2)$ & $28,6 \%(26)$ & $5,5 \%(5)$ & $1,1 \%(1)$ & $3,3 \%(3)$ & $40,7 \%(37)$ \\
Imposible & $0,0 \%(0)$ & $1,1 \%(1)$ & $6,6 \%(6)$ & $5,5 \%(5)$ & $2,2 \%(2)$ & $15,4 \%(14)$ \\
Contingente & $0,0 \%(0)$ & $5,5 \%(5)$ & $3,3 \%(3)$ & $0,0 \%(0)$ & $1,1 \%(1)$ & $9,9 \%(9)$ \\
Inevitable & $2,2 \%(2)$ & $3,3 \%(3)$ & $7,7 \%(7)$ & $13,2 \%(12)$ & $7,7 \%(7)$ & $34,1 \%(31)$ \\
Total & $4,4 \%(4)$ & $38,5 \%(35)$ & $23,1 \%(21)$ & $19,8 \%(18)$ & $14,3 \%(13)$ & $100,0 \%(91)$ \\
\hline
\end{tabular}

Nota: Las frecuencias absolutas se encuentran entre paréntesis junto a los porcentajes. Un total de 186 fueron empleadas para los análisis de fiabilidad. El acuerdo inter-codificadores fue de $\mathrm{K}=0,82$ para las formas modales, $\mathrm{K}=0,89$ para las razones y $\mathrm{K}=0,91$ para sujetos semánticos.

La tabla 2 muestra los estimadores correspondientes a las interacciones de más alto nivel de nuestro modelo. Los cinco efectos marginales y el resto de interacciones han sido omitidos debido al menor potencial analítico que presentan para el análisis del cambio de la modalidad lingüística.

Tabla 2. Estimaciones del Modelo Jerárquico Log-lineal de las interacciones entre las variables Forma Modal (m), Razón (r), Persona (p) y Tiempo Lineal (l)

\begin{tabular}{|c|c|c|c|}
\hline & Español & Miembro EU & Inmigrante \\
\hline & $\mathrm{m} \times \mathrm{r} \times \mathrm{p} \times 1$ & $\mathrm{~m} \times \mathrm{r} \times \mathrm{p} \times 1$ & $\mathrm{~m} \times \mathrm{r} \times \mathrm{p} \times 1$ \\
\hline \multicolumn{4}{|l|}{ Razón cultural } \\
\hline Posible & $0,194^{*}(0,111)$ & $-0,123(0,110)$ & $-0,039(0,060)$ \\
\hline Imposible & $0,008(0,031)$ & $-0,011(0,032)$ & $0,086(0,546)$ \\
\hline Contingente & $-0,128(0,299)$ & $0,230(1,467)$ & $0,330(0,888)$ \\
\hline Inevitable & $-0,015(0,028)$ & $0,021(0,031)$ & $0,003(0,053)$ \\
\hline \multicolumn{4}{|l|}{ Razón económica } \\
\hline Posible & $-0,001(0,024)$ & $0,035(0,028)$ & $-0,008(0,028)$ \\
\hline Imposible & $-0,015(0,026)$ & $-0,001(0,029)$ & $0,018(0,056)$ \\
\hline Contingente & $0,025(0,040)$ & $-0,080(0,060)$ & $0,068(0,121)$ \\
\hline Inevitable & $-0,003(0,020)$ & $-0,010(0,024)$ & $-0,006(0,033)$ \\
\hline \multicolumn{4}{|l|}{ Razón política } \\
\hline Posible & $0,039^{*}(0,020)$ & $-0,051^{*}(0,022)$ & $-0,034^{*}(0,033)$ \\
\hline Imposible & $0,021(0,018)$ & $-0,009(0,021)$ & $-0,033(0,031)$ \\
\hline Contingente & $0,007(0,038)$ & $0,027(0,054)$ & $-0,021(0,044)$ \\
\hline Inevitable & $-0,046^{*}(0,016)$ & $0,037^{*}(0,018)$ & $0,052(0,026)$ \\
\hline
\end{tabular}




\begin{tabular}{rrrr}
\hline & Español & Miembro EU & Inmigrante \\
\hline Razón de seguridad & $\mathrm{m} \times \mathrm{r} \times \mathrm{p} \times 1$ & $\mathrm{~m} \times \mathrm{r} \times \mathrm{p} \times 1$ & $\mathrm{~m} \times \mathrm{r} \times \mathrm{p} \times 1$ \\
\hline Posible & $-0,024(0,019)$ & $0,025^{*}(0,022)$ & $0,038(0,067)$ \\
Imposible & $-0,010(0,017)$ & $0,009(0,019)$ & $0,023(0,031)$ \\
Contingente & $0,173(43,694)$ & $0,001(19,222)$ & $0,315(21,323)$ \\
Inevitable & $0,036^{*}(0,015)$ & $-0,027(0,016)$ & $-0,033(0,028)$ \\
\hline Razón de bienestar & & & \\
\hline Posible & $-0,027(0,030)$ & $-0,443(0,476)$ & $0,085(0,043)$ \\
Imposible & $-0,024(0,036)$ & $0,073(0,071)$ & $0,005(0,043)$ \\
Contingente & $0,022(0,093)$ & $0,335(22,385)$ & $-0,016(0,099)$ \\
Inevitable & $0,014(0,023)$ & $0,011(0,033)$ & $-0,037^{*}(0,031)$ \\
\hline
\end{tabular}

Nota: Los errores de estimación entre paréntesis; *Interacciones significativas al nivel de $\mathrm{p} \leq 0,05$.

Como se puede apreciar en la tabla 2 (Estimaciones y error estándar del Modelo Jerárquico Log-lineal), se producen ciertos cambios en la modalidad del discurso de la inmigración de los periódicos de nuestro estudio. Lógicamente, debido a la amplitud de los resultados obtenidos para los efectos interactivos entre las variables en el modelo, en la tabla, sólo se presentaría la interacción de variables de más alto nivel: $\mathrm{m} \times \mathrm{r} \times \mathrm{p} \times 1$. Aquellas que en realidad permitían mostrar la existencia de variaciones de la gramática semántica (modalidad lingüística) en el tiempo.

En primer lugar, para sujetos semánticos españoles, se aprecia un incremento estadísticamente significativo en las formas modales que expresan inevitabilidad (deber, tener que, estar obligado a, etc.) en relación con razones de seguridad $(0,036)$. Esto significa que la seguridad se vuelve un asunto que «requiere» (modalidad de necesidad) la atención de los españoles a medida que la inmigración se va afianzando en nuestro país. Cuando hablamos de seguridad nos referimos fundamentalmente a la imagen de la inmigración como una amenaza. Por ejemplo, al aumento en la percepción del número de inmigrantes (asociado a la psicosis de la invasión) y la supuesta relación con el incremento de la delincuencia y la inseguridad. En la tabla 1, podemos apreciar la existencia de una relación significativa $(\mathrm{p} \leq 0,05)$ en la interacción de las tres variables principales a lo largo del tiempo (forma modal $[\mathrm{m}] \times$ razón $[\mathrm{r}] \times$ sujeto semántico $[\mathrm{p}] \times$ tiempo lineal [1]). Dicha relación, en consonancia a la gramática semántica generada para este estudio, viene a significar que: «hay ciertas RAZONES DE SEGURIDAD por las cuales algo es INEVITABLE para los ESPAÑOLES» (p. e. «por que los inmigrantes se encuentran relacionados con hechos delictivos [...] el gobierno debe actuar»). Una relación que 
se va incrementando con el paso del tiempo (ver gráfico 1), ya que claramente vemos que se produce un aumento paulatino en la probabilidad de aparición de esta tipología gramática a lo largo de dicho período temporal (1999-2008), sobre todo a partir de la mitad de la década. En efecto, debemos tener en cuenta que es a partir de estos años (2004-2006) cuando el discurso de la inmigración se vuelve más radical y reacio (Cea D’Ancona, 2007). De hecho, estos datos coinciden con la época en la que los medios se centraron en transmitir la cara más negativa de la inmigración (la imagen de las avalanchas, los asaltos a casas por bandas de Europa del Este, las bandas latinas, etc.). En definitiva, se produce un cambio en la modalidad lingüística de la inmigración que tiene una clara correspondencia con la imagen transmitida por los medios: la necesidad de soluciones ante la amenaza de la inmigración.

\section{Gráfico 1. Evolución de la modalidad del discurso de la inmigración para el período 1999-2008 (Sujeto semántico español)}

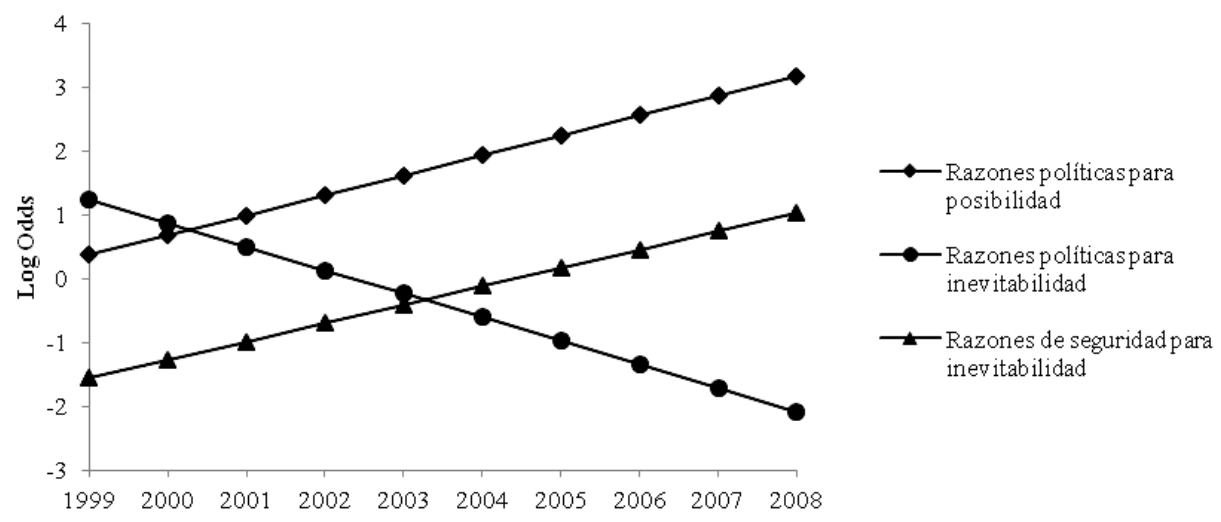

Año de publicación

Por otro lado, también podemos apreciar diferencias estadísticamente significativas $(\mathrm{P} \leq 0,05)$ en el paso de una «modalidad de necesidad» (expresada por formas modales de inevitabilidad) a otra «del logro» (expresada por modales de posibilidad: poder, ser capaz de, etc.) asociada a razones políticas. Así, lo que antes era «necesario» para los españoles por razones políticas deja de serlo $(-0,046)$, y se vuelve «posible» $(0,039)$. De acuerdo a nuestra gramática semántica esto significaría que «hay ciertas RAZONES POLÍTICAS por las cuales algo se vuelve POSIBLE para los ESPAN $O L E S$ » (p.e. «porque existen mecanismos político-legales para controlar la inmigración [...] el presidente del gobierno puede solucionar el problema de la inmigración») (ver gráfico 1).

Para sujetos semántico europeos, en cuestión de razones políticas, se produce un incremento en la probabilidad de aparición de este tipo de razones para una modalidad de inevitabilidad («modalidad de necesidad») y un descenso en la modalidad de posibilidad (poder, ser capaz de, etc.). Justamente al contrario 
que en caso de los sujetos semánticos españoles (ver gráfico 2). La gramática semántica sería la que sigue: «hay ciertas RAZONES POLÍTICAS por las cuales algo se vuelve INEVITABLE para los MIEMBROS-UE». Lo cual muestra una inversión en el discurso hacia uno u otros sujetos semánticos que comienza a darse a partir del año 2002, el momento en el que, a partir de las regularizaciones previas (Ley Orgánica 4/2000), la inmigración comienza a afianzarse como uno de los principales problemas de España. Un fenómeno que, en definitiva, da sentido a una inversión en la responsabilidad de los actores del discurso. Esto es, por ejemplo, para los actores políticos españoles la fuente del texto hace referencia a la posibilidad en su actuación (por ejemplo: «El gobierno puede, es capaz de...»), probablemente animando a la actuación de los mismos ante el asunto de la inmigración (la posibilidad para abordar el problema de cara a la búsqueda de soluciones). Para los actores políticos europeos se hace referencia a una modalidad de necesidad (inevitabilidad: Por razones políticas «la UE tiene que, debe, debería, está obligada a...»), un discurso que parece reclamar la atención de dichos sujetos ante el problema de la inmigración $(0,037)$. Es decir, se les invita a intervenir. Quizás por el mismo motivo que se invita a la actuación de los españoles por razones de seguridad frente a la amenaza de la inmigración.

\section{Gráfico 2. Evolución de la modalidad del discurso de la inmigración} para el período 1999-2008 (Sujeto semántico, UE)
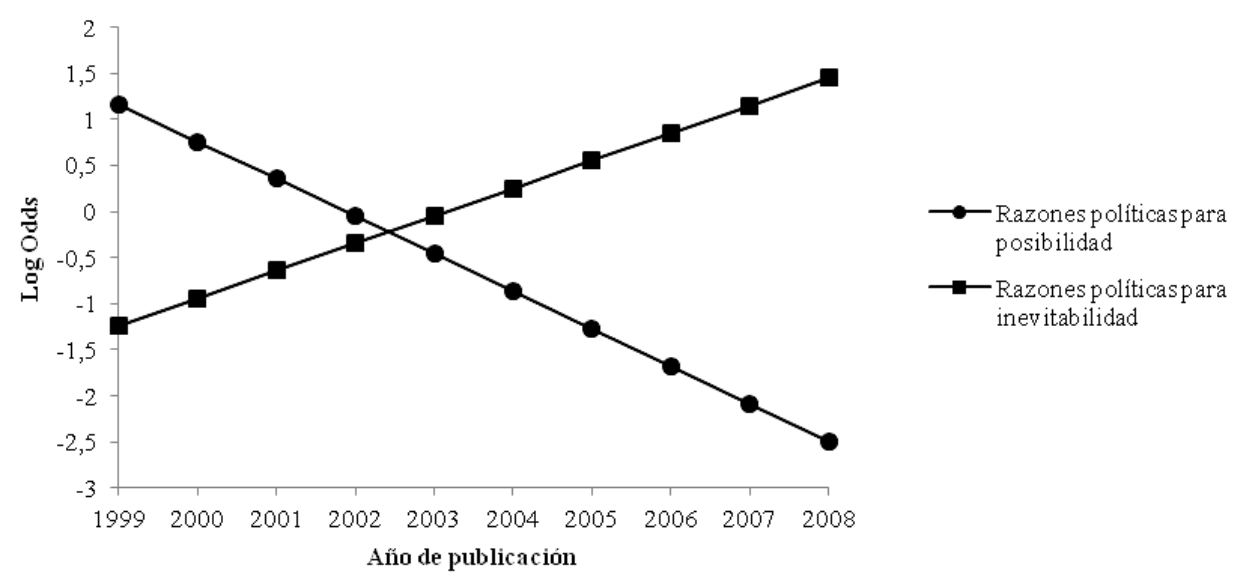

En última instancia, para los sujetos semánticos inmigrantes (o países emisores de inmigración), al igual que para los españoles, se aprecia un paulatino aumento en las razones políticas de cara a la posibilidad $(-0,034)$. Un discurso que invita a la actuación de los políticos de los países emisores (Marruecos, Senegal, etc.) a actuar frente al problema de la inmigración (ver gráfico 3). Asimismo, de manera acorde a este tipo de argumentos de posibilidad política se produce un incremento en la probabilidad de la aparición de razones 
de bienestar para una modalidad de necesidad $(-0,037)$. Esto es, «hay ciertas RAZONES DE BIENESTAR por las cuales algo se vuelve INEVITABLE para los INMIGRANTES». Razones de bienestar que fundamentalmente estarían referidas al bienestar (ayuda, asistencia, socorro, etc.) que deberían proporcionar los gobiernos de los países emisores hacia sus compatriotas.

Lógicamente, en nuestros análisis encontraríamos otros tipos de interacciones significativas de menor nivel, aunque también de menor poder explicativo. Así, por ejemplo, se apreciaba una disminución del discurso cultural (positivo) a lo largo del tiempo $(\mathrm{r} \times 1)$, un fenómeno que tiene relación con el propio aumento del discurso sobre política y seguridad en relación al tema de la inmigración como problema. También podemos encontrar interacciones significativas para razones de tipo económico $(\mathrm{m} \times \mathrm{r} \times \mathrm{p})$, aunque tampoco encontramos una pauta estable a nivel temporal.

Gráfico 3. Evolución de la modalidad del discurso de la inmigración para el período 1999-2008 (Sujeto semántico inmigrante)

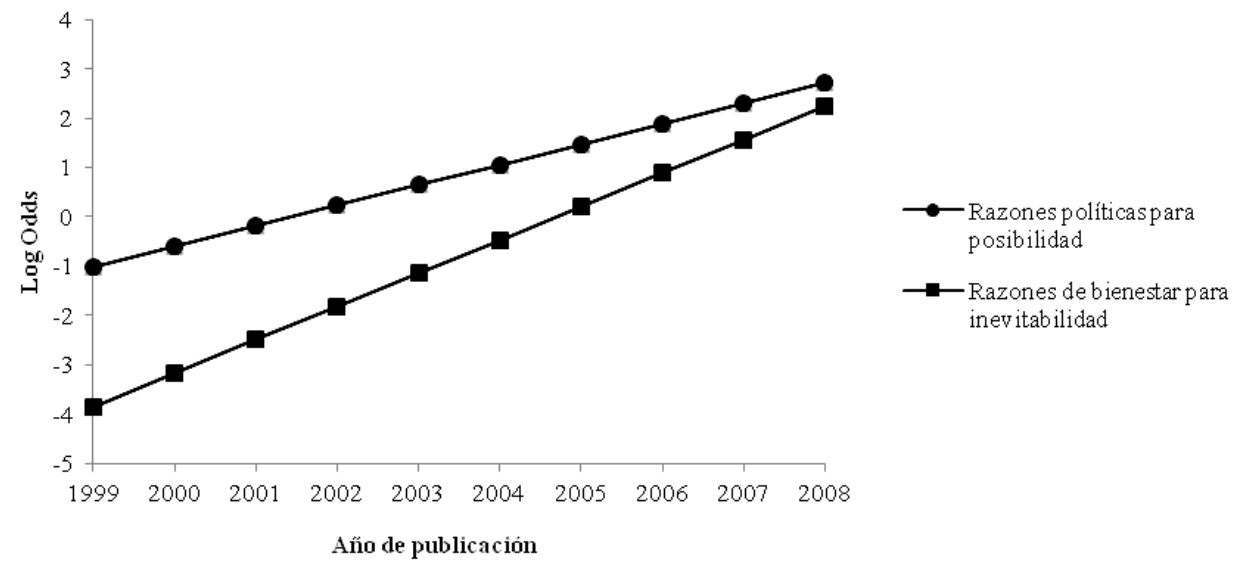

\section{CONCLUSIONES}

Los resultados de este trabajo muestran claros indicios de la dirección tomada por el discurso de los españoles hacia la inmigración de los últimos diez años. Aunque lógicamente no podamos extrapolar las conclusiones de este trabajo a la totalidad del discurso de la inmigración en nuestro país, puesto que no estamos tomando datos representativos del conjunto de medios de comunicación, si que podemos hacernos una idea del rumbo adoptado por dicho discurso. De hecho, el paulatino incremento de una «modalidad de la necesidad» ante razones de seguridad nos advierte del tipo de tratamiento que la inmigración ha recibido en los medios, habitualmente siendo catalogada como una amenaza para el mantenimiento del orden social. Un fenómeno que asimismo muestra 
una clara relación con la demanda de soluciones políticas que posibiliten («modalidad del logro») la erradicación de dicha amenaza. Del mismo modo que se invita a participar a los actores políticos europeos y extranjeros a colaborar en la solución de dicho problema.

A nivel metodológico, el presente estudio evidencia la utilidad de esta técnica de análisis. El empleo del denominado «modality analysis» sobre editoriales de inmigración en España permite confirmar un desplazamiento significativo en el discurso sobre este fenómeno hacia líneas argumentativas cada vez más problemáticas. Unas líneas discursivas que, muy probablemente, se encuentran asociadas con el aumento de las actitudes negativas hacia la inmigración de los últimos años. En definitiva, el presente trabajo representa un paso necesario hacia la comprensión de los posibles efectos del lenguaje sobre el condicionamiento de la opinión pública y, en última instancia, las dinámicas complejas de la acción social vinculadas a la (re)producción de actitudes hacia los inmigrantes.

A modo de limitación, debemos resaltar que sería necesario ampliar la muestra de diarios analizados para aumentar la representatividad estadística de los resultados. Asimismo, en los futuros estudios se deberían de tener en consideración las diferencias específicas entre los periódicos empleados, las cuales podrían aportar una información más concreta sobre, por ejemplo, el posicionamiento opinático-actitudinal de los mismos frente a las temáticas analizadas.

\section{BIBLIOGRAFIA}

AGRESTI, A. (1990) Categorical Data Analysis. New York: Wiley.

ÁLVAREZ-GÁLVEZ, J. (2009) «La representación mediática de la inmigración. Entre el encuadre y el estigma», Revista Migraciones Internacionales. Ministerio de Trabajo e Inmigración, n. ${ }^{\circ}$ 80: 61-80.

BALL-ROKEACH, S. J. (1985) «The origins of Individual Media-System Dependency: A sociological Framework». Communication Research, vol. 12, n. ${ }^{\circ} 4$, pp. 485-510.

BALL-ROKEACH, S. J, DEFLEUR, M. L. (1976) «A dependency model of massmedia effect». Communication Research, vol. 3, n. ${ }^{\circ}$ 1, pp. 3-21.

CASERO, A. (2006) «Discurso mediático, inmigración e ilegalidad: legitimando la exclusión a través de las noticias». Sitio web de la Fundación CIDOB.

CEA D'ANCONA, M. ${ }^{a}$ A. (2004) La activación de la xenofobía en España. ¿Qué miden las encuestas? Madrid, CIS/Siglo XXI, Monografía n. ${ }^{\circ} 210$.

CEA D'ANCONA, M. ' A. (2007) Inmigración, racismo y xenofobia en el nuevo contexto europeo. Madrid. Observatorio Español del Racismo y la Xenofobia (OBERA$\mathrm{XE})$. Ministerio de Trabajo y Asuntos Sociales.

CEA D'ANCONA, M. a A., VALLES, M. y ÁLVAREZ, J. (2006-2008) La medición de la xenofobia en la España de comienzos del siglo XXI: nuevos indicadores y diseños de encuesta para las políticas de integración de los inmigrantes. I+D MEC (ref. SEJ2005-00568). 
CEA D'ANCONA, M. ${ }^{a}$ A. y VALLES MARTÍNEZ, M. (2008) Evolución del racismo y la xenofobia en España. Madrid, Ministerio de Trabajo y Asuntos Sociales, Observatorio Español de Racismo y Xenofobia.

CEA D'ANCONA, M. a A. y VALLES MARTÍNEZ, M. (2009) Evolución del racismo y la xenofobia en España. Madrid, Ministerio de Trabajo y Asuntos Sociales, Observatorio Español de Racismo y Xenofobia.

CHECA, F. y ESCOBAR, P. (1996) Inmigrantes africanos en la prensa diaria almeriense (1990-1994). De invasores e invadidos. En Kaplan, A. (Coord.) Procesos migratorios y relaciones interétnicas (pp. 145-164). Zaragoza. Instituto Aragonés de Antropología.

D'ADAMO, O., GARCÍA BEAUDOUX, V., y FREIDENBERG, F. (2000) Medios de comunicación de masas, efectos políticos y opinión pública. ¿Una imagen vale más que mil palabras? Buenos Aires: Editorial de Belgrano.

D'ADAMO, O., GARCÍA BEAUDOUX, V. y FREIDENBERG, F. (2007) Medios de Comunicación y Opinión Pública. Madrid: McGraw Hill.

DAVIDIAN, M. y GILTINAN, D. M. (1995) Non-linear Models for Repeated Measurement Data. New York: Chapman and Hall.

DIEZ NICOLAS, J. y RAMÍREZ LAFITA, M.J. (2001) La inmigración en España: una década de investigaciones. Colección Observatorio Permanente de la Inmigración. Madrid. Ministerio de Trabajo y Asuntos Sociales.

GOFFMAN, E. (1974) Frame analysis: An essay on the organization of experience. Cambridge, MA: Harvard University Press.

GRANADOS, A. (1998) La imagen del inmigrante extranjero en la prensa española: ABC, Diario 16, El Mundo y El País (1985-1992).Tesis Doctoral. Universidad de Granada.

IGARTUA, J. J., MUÑIZ, C., CALVO, P., OTERO, J. A., y MERCHÁN, J. (2005) «La imagen de la inmigración en la prensa y en la televisión. Aproximaciones empíricas desde la teoría del framing». En Sabucedo, J.M., Romay, J. y López-Cordón, A. (comps.) Psicología, cultura, inmigración y comunicación social. Madrid. Ed. Biblioteca Nueva: 239-246.

LORITE, N. (dir.) (2004) Tratamiento informativo de la inmigración en España 2002. Madrid: Instituto de Migraciones y Servicios Sociales.

LUHMANN, N. (2000) La realidad de los medios de masas. Barcelona. Anthropos, 2000.

MCCOMBS, M. (2004) Setting The Agenda: The Mass Media And Public Opinion. England, Polity Press. UK: Cambridge.

MCLUHAN, M. (1964) Understanding Media: The Extensions of Man. New York. McGraw-Hill.

PINHEIRO, J. C. y BATES, D. M. (1995) «Approximations to the Log-Likelihood Function in the Nonlinear Mixed-Effects Model», Journal of Computational and Graphical Statistics, 4: 12-35.

ROBERTS, C. (2000) «A Conceptual Framework for Quantitative Text Analysis». Quality and Quantity 34 (3, August): 259-274.

ROBERTS, C. W. (2008) 'The' Fifth Modality: On Languages that Shape Our Motivations and Cultures. Leiden: Brill.

ROBERTS, C. W., ZUELL, C., LANDMANN, J., y WANG, Y. (2010) «Modality analysis: A semantic grammar for imputations of intentionality in texts». Quality \& Quantity 44: 239-257. 
ROBERTS, C. W., POPPING, R., y PAN, Y (2009) «Modalities of Democratic Transformation: Forms of Public Discourse in Hungary's Largest Newspaper, 1990-97». International Sociology, 24: 498-525.

RODRIGO ALSINA, M. (1999) Comunicación intercultural. Barcelona. Antropos.

SAID, E. (1990) Orientalismo, Madrid, Ediciones Libertarias.

SANTAMARÍA, E. (2002) «Inmigración y barbarie. La construcción social y política del inmigrante como amenaza». Papers, 66, 51-58.

VALLES, M. S.; CEA D'ANCONA, M. A.; e IZQUIERDO, A. (1999) Las encuestas sobre inmigración en España y en Europa. Madrid, IMSERSO.

VAN DIJK, T. A. (1991) Racism and the press, London; New York : Routledge, 1991.

VAN DIJK, T. A. (1993) Elite Discourse and Racism Newbury Park: Sage, in press.

VAN DIJK, T. A. (1997) Racismo y análisis crítico de los medios. Barcelona: Paidós.

VAN DIJK, T. A. (2003) Dominación étnica y racismo discursivo en España y América Latina. Barcelona: Gedisa.

VECINA, C. (2012) «Un estudio sobre las Representaciones Sociales de la inmigración en la prensa y en una revista de barrio». Revista Electrónica de Investigación y Docencia (REID). Núm. monográfico 2, 32-55.

VONESH, E. F. y CHINCHILLI, V. M. (1997) Linear and Nonlinear Models for the Analysis of Repeated Measurements. New York: Marcel Dekker. 